\title{
Microwave detection of buried mines using non-contact, synthetic near-field focusing
}

\author{
R.Benjamin, I.J.Craddock, G.S.Hilton, S.Litobarski, E.McCutcheon, R.Nilavalan and G.N.Crisp
}

\begin{abstract}
Existing ground penetrating radars (GPR) are limited in their 3-D resolution. For the detection of buried land-mines, their performance is also seriously restricted by 'clutter'. Recent work by the authors has concentrated on removing these limitations by employing multi-static synthetic focusing from a 2-D real aperture. This contribution presents this novel concept, describes the proposed implementation, examines the influence of clutter and of various ground features on the system's performance, and discusses such practicalities as digitisation and timesharing of a single transmitter and receiver. Experimental results from a variety of scenarios are presented.
\end{abstract}

\section{Introduction}

\subsection{Requirement}

While accurate figures are impossible to obtain, it has becn claimed [1] that over 100000000 land-mines are laid in over 60 countries, and new ones arc still being laid faster than old ones are clcared. Detection of these mines still depends largely on manual probing or, if the nature of the mines and environment permits, metal-detectors or groundpenetrating radars (GPR).

A variety of land-mine detection sensors have been developed [2,3] and, as any GPR system will find it difficult to distinguish between a mine and a clutter object of similar size, shape and reflectivity, it is widely envisaged that a successful system will require a fusion of data from a number of different sensor types [4]. In offering the potential of both rapid search and significant ground penetration [5], a GPR sensor has potentially the most important part to play. However, in order to realise this potential in attenuating soil containing significant clutter, major improvements to GPR are needed [6]. This contribution reports a new, vehicle-mounted, GPR that goes a long way to meet these requirements.

This paper does not attempt a rigorous analysis of clutter and its effect on this radar system but instead concentrates on the engineering calculations used to design the system, and on the operational system envisaged. Numerical results confirm the predicted resolution of the system and previously unpublished practical results for small plastic

(C) Crown copyright 2001

Reproduced by permission of the Controller of Her Majesty's Stationery Office

IEE Proceedings online no. 20010421

DOI: $10.1049 /$ ip-rsn:20010421

Paper first received 8th November 2000 and in revised form 8th March 2001

R. Benjamin, I.J. Craddock, G.S. IIilton, S. Litobarski, E. McCutcheon and R. Nilavalan are with the Centre for Communications Research, Merchant Venturers Building, University of Bristol, Woodland Road, Bristol BS8 1UB, UK

G.N. Crisp is with DERA Malvern, St Andrews Road, Malvem WR14 3PS, UK anti-personnel (AP) mines in diverse, realistic, outdoor environments clcarly demonstrate its capability.

\subsection{Real-aperture synthetically organised radar (RASOR)}

In-contact operation of GPR avoids ground reflection and refraction. However, even if the ground was sufficiently smooth and clear to permit in-contact operation, there would be a very high risk of setting off trigger devices. The authors' work has therefore concentrated on noncontact operation with a 2-D planar in-air antenna array.

Bristol's RASOR system [7-10] permits optimum detection, in terms of signal to clutter ratio, of buried objects by GPR: all elements of the array transmit a broadband pulse (two cycles of a $1 \mathrm{GHz}$ carricr in our experimental implementation) in turn; all elements sharing any operationally relevant 3 -D field of view with the current transmit element then record the received transient signal. By predicting the path delay from the transmit antenna $A$ via any desired resolution cell $C$ to any receiving antenna $B$ (Fig. 1), it is then possible to retrospectively extract and time-align all the signals from the resolution cell. The reflected energy associated with this resolution cell is therefore:

$$
V=\int_{0}^{\tau}\left(\sum_{i=1}^{N} w_{i} U_{i}\left(t-T_{i}\right)\right)^{2} d t
$$

where $U_{i}(t)$ is the signal received from path $i, N$ is the number of paths associated with the resolution cell, $w_{i}$ is a weighting factor associated with the attenuation, spreading losses and susceptibility to clutter along path $i, T_{i}$ is the

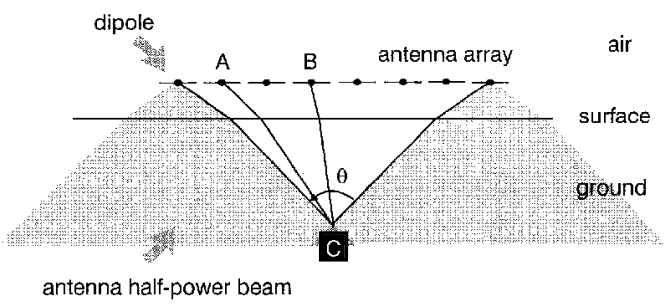

Fig. 1 Transmit antema $A$, receive antenna $B$, resolution cell $C$ 
path delay associated with the trip to and back from the resolution cell and $\tau$ is the transmit pulse width. Knowledge of the path delay requires estimation of the in-ground propagation velocity and will ultimately fail if the ground is highly inhomogenous (Section 2.7 describes an alternative). As shown by eqn. 1 above, the return associated with the resolution cell is computed by integrating the power in the sum of time-aligned and weighted signals, over a window corresponding to the transmit pulse width. This process is repeated for all resolution cells.

It is important to note that this is a fundamentally different mode of opcration to the well-known synthetic aperture radar (SAR) technique. RASOR generates distinct signals from all possible combinations of transmit and receive elements forming a fully populated array (quite unlike [11], for example). In contrast, SAR relies upon vehicle movement to generate a complete array aperture. This permits the use of an array of smaller physical size, but consequentially loses the valuable information from bistatic paths. The information from the large number of independent bistatic paths gives RASOR far greater immunity to clutter and, as explained in Section 3.3.2, a large, fully populated antenna array is not a great problem for a vehicle-mounted system.

Fig. 1 shows in one dimension the set of antenna elements that can observe a given resolution cell in the ground, including the effect of refraction, but, for simplicity (as elsewhere in this contribution) representing the antenna element pattern by a uniform mainlobe, without a 'skirt' or sidelobes. With $N$ elements, there are $N^{2}$ possible bistatic paths, however duplexing problems make it unattractive to receive on the transmitting element and, since the total bistatic path would be the same if the transmit and receive function were interchanged, therc are in fact $N(N-1) / 2$ distinct paths per resolution cell. The ability to identify the individual signals corresponding to these distinct paths offers important new signal-processing options.

\subsection{Operational details}

A high operating frequency permits a high bandwidth and hence high resolution, good distinction of shallowly-buried targets from surface reflections, good back-scatter from small targets, and high processing gain for a given size of array. A lower frequency, however, offers good ground penetration and greater tolerance of surface roughness. The illustrative sample design suggested here, based closely upon our current experimental system (but using a larger array) compromises on a centre frequency of $1 \mathrm{GHz}$. As shown in Section 4, this frequency gives just sufficient resolution for the detection and localisation of small plastic $\mathrm{AP}$ mines and therefore provides good immunity against significantly smaller clutter objects (whose scattering cross section reduces according to the fourth power of the object's electrical size).

The suggested design uses a horizontal rectangular array at $0.75 \lambda_{\mathrm{a}}$ spacing, where $\lambda_{\mathrm{a}}$ is the pulse's centre-frequency wavelength in air, for reduced mutual coupling. In nearfield focusing, this spacing does not generate diffraction lobes.

An array-to-ground spacing of $2 \lambda_{\mathrm{a}}$ and an element beam-width of $\pm 45^{\circ}$ implies an array width of $\pm 2 \lambda_{a}$, merely to focus onto the surface centre-line. To cover the in-soil divergence of the beam, a further $\pm 2 \lambda_{\mathrm{a}}$ is provided, sufficient to maintain full gain and resolution for virtually any practical refractive index and burial depth, e.g. $66 \mathrm{~cm}$ at a refractive index of 5 , with $\lambda_{\mathrm{a}}=30 \mathrm{~cm}$. This requires an

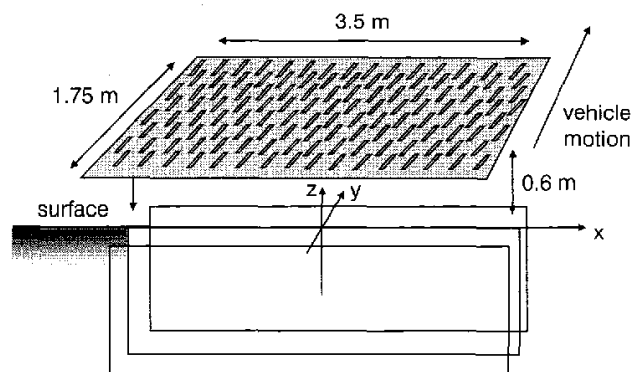

Fig. 2 Full-sized array and stack of three scanned planes

array width of 8 elements. To reduce mutual coupling further, these are arranged in two interleaved sets of 4 coplanar elements, spaced at $1.5 \lambda_{\mathrm{a}}$ centre-to-centre, separated along the length of the array by $0.375 \lambda_{a}$, maintaining an effective $0.75 \lambda_{a}$ length-wise spacing (see Fig. 2). Naming each such set of 4 elements a 'column', 18 columns cover a sweep-width of $2 \mathrm{~m}$. To focus with full gain onto the extremities of this sweep, a further 7 columns are placed beyond each end, giving 32 columns and a size of approximately $3.5 \mathrm{~m}$ by $1.75 \mathrm{~m}$ (32 columns of 4 elements, 128 elements in total).

Through application of the focusing process described in Section 1.2, the array generates a picture of a $2 \mathrm{~m}$ wide, $10 \mathrm{~cm}$ thick vertical slice underneath its major axis. A $30 \mathrm{~cm}$ thick stack of three such slices is formed, and this is swept forward by the motion of the carrying vehicle, as in Fig. 2. A set of three simple ultrasound or infrared distance scnsors record the mean height and orientation of the array to the ground. Simple mechanical actuators control the approximate position of the array and any departures from the ideal array position will be allowed for in processing.

From antenna reciprocity $[12]$ it can be shown that the resolution is determined by the wavelength $\lambda$ and the convergence angle $\theta$ (Fig. 1). From [12] the lateral resolution is $\lambda_{\mathrm{g}} / 2 \theta$. In the ground, the convergence angle $\theta$ is reduced by refraction, approximately as $1 / \sqrt{ } \varepsilon$ (where $\varepsilon$ is the dielectric constant) however, since the wavelength in the ground $\lambda_{\mathrm{g}}$ is similarly reduced, the lateral resolution is virtually unchanged.

The depth of focus, $2 \lambda_{\mathrm{g}} / \theta^{2}$ [12], however increases (is degraded) as $\sqrt{\varepsilon}$, but only up to the point where it is limited by the pulse length in the ground, $n \lambda_{\mathrm{a}} /(2 \sqrt{ } \varepsilon)$, where $n$ is the number of cycles in the pulse length, 2 in our design, giving a vertical resolution of $0.5 \%_{\mathrm{a}}$ for $\varepsilon=4$.

\section{Performance-limiting factors}

This Section describes the various factors that limit the performance of the GPR array and the steps taken to overcome them.

\subsection{Thermal noise limits}

Any 3-D resolution cell in the ground will be within view of 64 elements of our array, albeit with the gain of the outer elements reduced for shallower burial depths, but increasing to full gain with increasing burial depth and refractive index. This gives rise to $64 \times(64-1) / 2=2016$ distinct paths. Combining these signals coherently gives a nominal processing gain of $33 \mathrm{~dB}$. A conventional GPR of the same power could achieve the same gain against thermal noise, but not against clutter, if it emitted sufficiently many pulses and integrated their echo signals coherently. 
Table 1: Maximum detectable depths of burial

\begin{tabular}{lrcc}
\hline Moisture (\%) & 10 & 30 & 50 \\
\hline$\varepsilon_{\mathrm{r}}$ & 5 & 17 & 38 \\
$\varepsilon_{\mathrm{rr}}$ & 1 & 3.45 & 6.6 \\
Burial depth $(\mathrm{cm})$ & 100 & 50 & 35 \\
\hline
\end{tabular}

Table 1 presents theoretical results for the effect of soil moisture content on the detection of a $4 \mathrm{~cm} \times 4 \mathrm{~cm}$ metal plate. These figures are for $1 \mathrm{~W}$ of $\mathrm{RF}$ power at $1 \mathrm{GHz}$, using data from [5] and elsewhere, showing that both attenuation and refractive index are determined predominantly by the moisture content of the soil. Although neardistilled water causes minimal loss or change of refractive index, and there are also some rare, freak soils which are heavily attenuating even when dry, and others which have a large refractive index with little attenuation.

A plastic mine has a greater contrast to wet soil, giving an enhanced echo to set against increased path loss. Furthermore, the shortening of the in-ground wavelength, due to the high refractive index normally associated with high attenuation, considerably enhances the echo strength of small AP mines. Consequently, the system will not normally be noisc-limited. The main challenge is instead to overcome clutter.

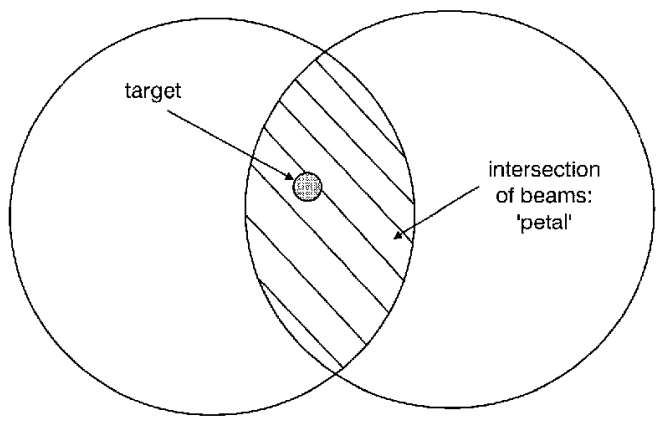

Fig. 3 Clutter petal (plan view)

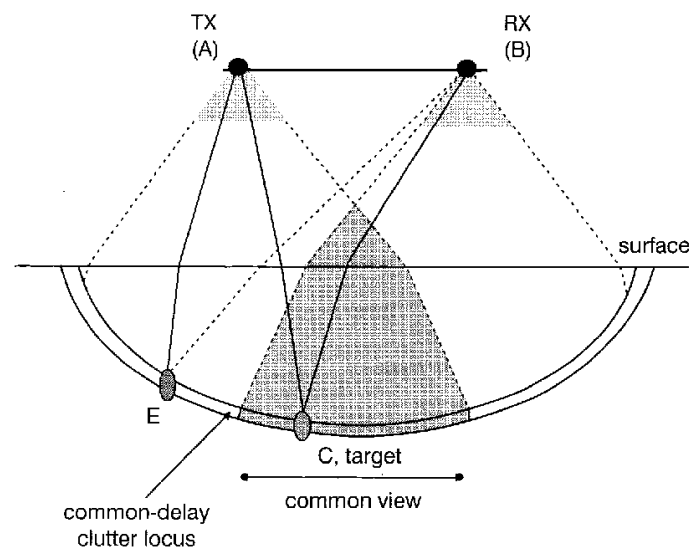

Fig. 4 Clutter petal (elevation)

\subsection{Clutter weighting}

2.3.1 Petal weighting: It has been established by modelling that the two-way combined spreading and attenuation loss to any part of a given petal is virtually constant. Therefore, if the local clutter is quasi-uniformly distributed, the signal/clutter ratio is inversely proportional to $A_{C}$, the area of the petal (see Fig. 3) and, for any unknown clutter distribution, the probable signal to clutter ratio is similarly proportional to $1 / A_{c}$.

To minimise the effect of such non-coherent volume clutter, each path signal should then be weighted inversely to $A_{c} . A_{c}$ is approximately proportional to the product of its width, $2 W$ and its length $2 H$. These parameters are determined by $R$, the beam radius at the target depth and $S$, half the separation of the antenna elements, yielding:

$$
A_{c} \approx k_{p} W H=k_{p}(R-S) \sqrt{R^{2}-S^{2}}
$$

where $k_{p}$ is a constant.

Any spot-focused radar operation implicitly involves the path between each segment (or element) of the transmitting antenna system, via the focal spot, to each segment (or element) of the receiving antenna system. However, only RASOR can isolate the distinct signal contributions from the $N(N-1) / 2$ distinct two-way paths between $N$ antenna elements 'seeing' the target. It thus reveals the nature of tho focusing process, which, in simplificd form, may be regarded as the intersection of the resultant $N(N-1) / 2$ petals (as shown schematically in Fig. 5). By generating and recording these as distinct contributions RASOR is (uniquely) able to weight them selectively.

2.3.2 Path-loss equalisation: If all paths are to make their optimum contribution vis-à-vis clutter, any differ- 

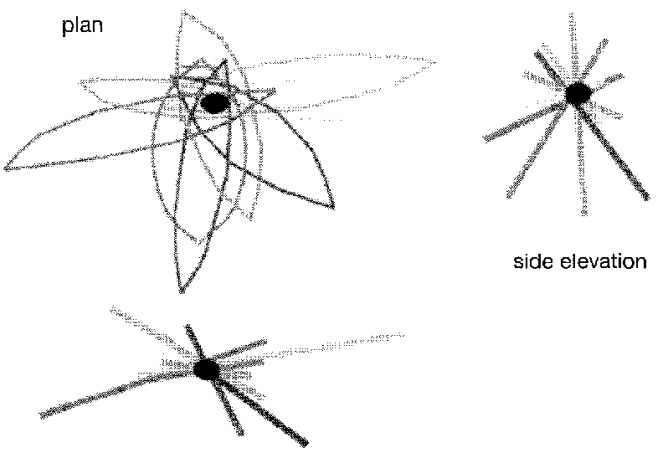

side elevation

$$
\text { front elevation }
$$

Fig. 5 Schentatic representation of definition of focal spot as common element of multiple overlapping 'petals' of diverse size, shape and orientation

ences in their individual losses (due to spreading losses in air and soil, incidence-angle dependent air-ground interface losses and attenuation in the soil) should be equalised, prior to petal weighting.

The in-soil attenuation is negligible for very shallowly buried targets. The differential in-soil attenuation between different paths is also low for most high-loss soil, since this generally implies a high refractive index, and hence a very narrow convergence angle. Normally, only deeply buried targets in moderate-loss soil exhibit any significant differential in soil attenuation.

As discussed in Section 3.3.1, the signal is amplified by the predicted (primarily depth dependent) loss before digitisation in order to match the dynamic range of the digitiser.

2.3.3 Surface clutter: The detection of mines buried deeply in an attenuating medium might well be impaired by surface clutter, which escapes this attenuation. However, surface clutter can compete with the wanted target from a given two-way path only within the surface area defined by the overlap of:

- the 'race-track' shaped locus (Fig. 6) whose outer and inner perimeters correspond to a path delay equal to that from $A$ to $B$ via cell $C$, plus or minus half the pulse length (in air), respectively.

- the (in air) beam widths of both the transmit and receive elements.

Furthermore, any specular reflection from the transmit path will point well away from the receive element, and so clutter can only arise from diffuse scattering. In practice, as shown in Fig. 6, deeply buried objects produce no significant antenna-beam overlap and so do not suffer seriously from surface clutter.

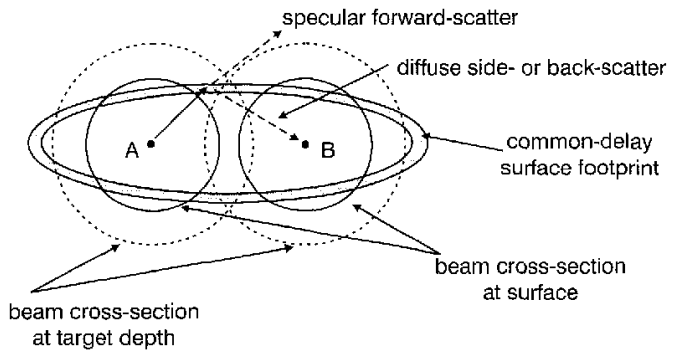

Fig. 6 Plan view of surface clutter locus
For very shallowly buried objects, the inner perimeter of the 'race-track' locus shrinks and can disappear completely, and since, for such targets, there is little further divergence of the beams after ground-entry, there is substantial overlap between the beams, especially for closely spaced antenna elements. With unfavourably concave ground, some element pairs could then even experience localised specular reflection. Otherwise however, only diffuse scattcr is relcvant.

Specular returns from surface clutter generally arise from a slightly shorter path than that via the target resolution cell, hence they have only limited, if any, time overlap with the target signal. Given an appropriate means of cancelling the surface reflection (see the following Section), surface clutter is thcrefore a manageable problem, as evidenced by the results presented in Section 4 .

2.3.4 Cancellation of surface clutter and mutual coupling: Very shallowly buricd targets are difficult to distinguish from surface reflections. However the surface characteristics are correlated over an area substantially larger than the resolution cell. Hence, in the absence of a target, the coupling between an antenna in position $\{x, y\}$ and one at $\{(x+\Delta x),(y+\Delta y)\}$ is identical to that for any laterally displaced pair $\{(x+p),(y+q)\}$ and $\{(x+p+\Delta x),(y+q+\Delta y)\}$, and also for their mirror images, where the signs of $\Delta x$ and/or $\Delta y$ are reversed.

For each such set of generic pair spacings in the array, a two-dimensional, complex (amplitude and phase) histogram of surface reflections can therefore be formed:

- When the histogram contains a well-defined peak, this provides a good estimate of the relevant surface reflection. If there are also some significant extraneous points, these can be assumed to be due to surface objects or other anomalies, and hence disregarded.

- When the histogram has a well-defined amplitude peak, but the phase yields no clearly identifiable peak, noncoherent clutter cancellation may be used, as discussed in Section 2.5 .

- If the histogram has a well-defined phase peak but none in amplitude, coherent subtraction of the mean amplitude, at the given phase, should still give some net benefit.

Unwanted couplings within the array itself are separately recorded in an anechoic environment and then cancelled in all measurements.

\subsection{Surface roughness}

If the amplitude of surface roughness is small compared to the wavelength in air, and its mean correlation distance is not much more than the wavelength, the surface gives rise to quite strong specular scattering, together with relatively weak diffuse back-scatter. If both the amplitude and mean correlation distance of the surface texture were comparable to, or larger than, the wavelength, it would give rise to strong diffuse scattering, and its 'prism' effect would also modify the directional distribution of the radiation in the soil. However, so coarse a texture is not very likely at wavelengths of $30 \mathrm{~cm}$ in air, except for ploughed fields and the like.

In the limit, surface roughness could prevent coherent processing if the phase variations, within the surface footprint of the set of paths converging onto a given resolution cell, due to 'rays' passing through soil rather than air (or vice versa) is substantially greater than $\pm \pi / 2$. 


\subsection{Non-coherent processing}

Even when the surface is too irregular or the refractive index of the ground is too inhomogeneous to permit coherent processing, there is normally a locally reasonably uniform volume clutter density $\rho_{c}$, whose value can be inferred from neighbouring resolution cells. Since the thickness of all the clutter petals is determined by the pulse length in the medium, and is thus common, the clutter power, from any given two-way path, will be proportional to $\rho_{c} A_{c}$, where $A_{c}$ is the relevant petal area.

The presence of a target signal will either increase or decrease the magnitude of the clutter, but only rarely leave it unchanged. Hence the modulus of the difference between the observed signal and the predicted clutter is a noncoherent signal component due to the target. These noncoherent single-path signals can be weighted, processed and combined in the normal way, but the resulting $\mathrm{dB}$ gain will be rather less than half that for coherent processing.

\subsection{Macro-scale ground features}

Likely macro-scale ground features are 2-D gradients in otherwise level ground (or in the positioning of the array) and concave or convex surface curvaturc.

Here, again, the effect is due to the difference in phase delay arising when an increment of pathlength (in the set of paths converging onto a given resolution cell) assumed to be in air, is actually in soil, or vice versa.

Curved ground acts like a lens, producing a small error in the target's apparent depth. High refractive index reduces the in-ground convergence angle and hence the surface footprint. This more than cancels the impact of the higher refractive index of the 'lens', and so reduces the effect. It is shown in Section 4 that realistic ground features only slightly degrade the focusing performance.

There will, however, be a range of scenarios, such as broken rocky ground or ruined buildings which are not amenable to our system, but these are also scenarios not generally accessible to any vehicle-mounted system.

\subsection{Stratified ground}

Analysis and finite difference time domain (FDTD) modelling show that strata interfaccs with refractive-index ratios up to $\sim 1.5$ have negligible effect. However, major discontinuities, say interfaces with refractive-index ratios $>4$, produce significant reflections. Their effect is similar to that of volume clutter, and the RASOR system overcomes it quite effectively.

When the strata are horizontally uniform, the effect is automatically cancelled by the 'surface-clutter' cancellation process of Section 2.3.4.

\subsection{Reverberation}

Multiple 'bounces' between the antenna array and surface could impair the detection of targets at the corresponding two-way path delays. This has been obviated by coating the back-plane of the antenna array in radar absorbing material. This also widens the array bandwidth from $40 \%$ to $78 \%$ [12] and also helps screen the system from external radio frequency interference (RFI). RFI has consequently never been a problem in any of our measurement activities, at a range of sites.

\section{Operational issues}

While considerable thought has been given to the practicalities of an operational system, space constraints permit only a brief discussion of some of the issues involved.

\subsection{Search rate}

When advancing into an uncleared area, the array would bc mounted on a boom ahead of the vehicle and a swathe $2 \mathrm{~m}$ wide would be cleared. The vehicle could well be immune to anti-personnel mines. If anti-tank (AT) mines have to be faced, its speed would be limited by the need to stop before it is over a mine. A boom extension of $5 \mathrm{~m}$, and a braking rate of $0.25 \mathrm{G}$, would permit an upper-limit speed of $18 \mathrm{~km} / \mathrm{h}$ (assuming automatic warning and application of the brakes). A tracked vehicle with a $10 \mathrm{~m}$ boom and a braking rate of $1 \mathrm{G}$ would permit a speed of $50 \mathrm{~km} / \mathrm{h}$, and this speed could also be used freely by a vehicle with a laterally projecting array, to extend the periphery of a cleared area, or by a vehicle exerting insufficient ground pressure to trigger an AT mine.

The overall mine clearance rate is however likely to be determined by the time taken to respond to the putative mines discovered, be they real or false alarms. Hence a more modest speed $v$ of $5 \mathrm{~m} / \mathrm{s}(18 \mathrm{~km} / \mathrm{h}$, i.e. $11.2 \mathrm{~m}$.p.h. $)$ is assumed below.

\subsection{Effect of vehicle motion}

A $30 \mathrm{~cm}$ advance of the vehicle, in the time taken to collect the data to generate a $30 \mathrm{~cm}$ stack of three $10 \mathrm{~cm}$ thick, $2 \mathrm{~m}$ wide vertical slices, is equivalent to a $8.6^{\circ}$ right-to-left skew of these vertical slices. Since data collection sweeps from left to right across the array (see Section 3.3.2), allowing for this tilt compensates for vehicle motion and so maintains data coherence. A vehicle speed of $v=5 \mathrm{~m} / \mathrm{s}$ allows $60 \mathrm{~ms}$ to collect one such three-slice data 'frame', in parallel with processing the data from the preceding frame.

\subsection{Data collection and recording requirements}

Assuming a maximum depth of burial entailing sixteen in-soil wavelengths for the two-way path, it is proposed to record this as 48 samples, to enhance the resolution and to comfortably exceed the Nyquist limit. With the RFswitching architecture proposed (which, for design simplicity, does not use all possible transmit-receive pairs) the total number of relevant paths within the array can be shown to be 5760 . Hence only $48 \times 5760=276480$ samples need to be recorded. However, the speed and dynamic range of digitisation would stretch the current 'state-of-the art', and the cost of 128 receivers would be quite high. The following two Sections show how these problems can be solved.

3.3.1 Time-dependent gain: 12-bit ( $72 \mathrm{~dB}$ ) quantisation would be ample to extract the wanted signals in the presence of clutter (or noise). However, the two-way attenuation to a deeply buried target might add up to $60 \mathrm{~dB}$. Fortunately, all the paths with a common roundtrip path delay between a given pair of elements have closely identical path losses. Hence, for known soil attenuation, the pre-digitisation amplifier gain can be adjusted, in 1-bit steps, to match the dynamic range to the A-to-D converter.

3.3.2 Time sharing: For optimum use of transmit power, the transmission from any element would be 
received concurrently by all other elements. However, time sharing provides a more economical solution. If the 5760 discrete paths are handled as separate transmissions and receptions, this gives $60 \mathrm{~ms} \div 5760=10.4 \mu \mathrm{s}$ per element pairing. Thus, it is possible to time-share one transmitter, one receiver, one path-loss compensator, and one block of 48 sample-and-hold circuits between all pairings. Allowing $0.4 \mu \mathrm{s}$ for switching to the correct elements, and for transmission and reception, $10 \mu \mathrm{s}$ would be available for digitising the 48 sample-and-hold outputs. This could be done sequentially by a single time-shared 12-bit digitiser (or by a small number of slower but cheaper digitisers).

The switching sequence is designed so that the set of resolution cells irradiated precesses as smoothly as possible from right to left across the vertical slice generated.

The $10.4 \mu$ s per antenna pairing would just permit one transmission from a given elcment to a given receiving element to be replaced by 48 such transmissions, spread cqually over the $10.4 \mu \mathrm{s}$ available [13], so that a single sample-and-hold circuit can also be time-shared. While this would use RF power very inefficiently, it clearly is the cheapest and easicst implementation of the basic scheme.

\section{Verification and status}

\subsection{Initial experiments and validation}

Initial validation of the system was by FDTD simulation [14] and then by sandbox experiments with metal and plastic targets (including clutter) $[9,10]$. In all cases, antenna elements were a printed bow-tie dipole design [15].

Early synthetic focusing experiments used an 8-element linear array, of length $l=1260 \mathrm{~mm}$, operating at a centrefrequency of $1 \mathrm{GHz}$, and a height $h=600 \mathrm{~mm}$ above the ground. This array is too small to achieve the resolution and anti-clutter performance needed, however its small size permits theoretical predictions, full-wave FDTD modelling and measurements to be compared.

These initial experiments considered a metallic target buried in sand (relative permittivity $\varepsilon_{\mathrm{r}}=3.2$ ) at a depth $d$ of $200 \mathrm{~mm}$. Applying Snell's law, in order to determine the convergence angle $\theta$ at the target produced by this aperture, yields $\theta=0.78$ radians $\left(44.7^{\circ}\right)$. From Section 1.3 , the lateral resolution for this array should therefore be:

$$
\frac{\lambda_{\mathrm{g}}}{2 \theta}=\frac{300 / \sqrt{3.2}}{2(0.78)}=107 \mathrm{~mm}
$$

The results from both FDTD and measurement presented in Fig. 7 clearly show the target location (buried at a lateral displacement of $780 \mathrm{~mm}$ ) and yicld a resolution of $115 \mathrm{~mm}$. This figure is in good agreement with the value of $107 \mathrm{~mm}$ derived above for this small array.

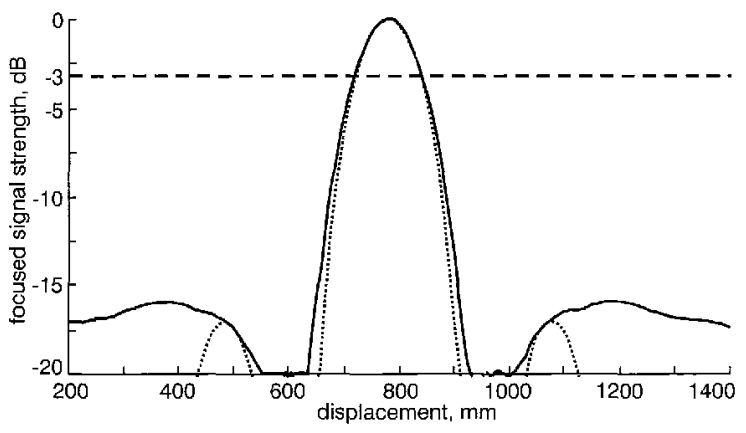

Fig. 7 Lateral focusing through target location
Table 2: Summary of focusing degradation for different surface types

\begin{tabular}{llll}
\hline Surface type & $\begin{array}{l}40 \% \\
\text { slope }\end{array}$ & $\begin{array}{l}\text { Convex } \\
(25 \mathrm{~cm} \text { high })\end{array}$ & $\begin{array}{l}\text { Concave } \\
(25 \mathrm{~cm} \text { deep })\end{array}$ \\
\hline $\begin{array}{l}\text { Change in processing } \\
\text { gain (dB) }\end{array}$ & -4.3 & +0.002 & -0.7 \\
$\begin{array}{l}\text { Target location } \\
\text { error (mm) }\end{array}$ & 50 & 25 & Negligible \\
$\begin{array}{l}\text { Horizontal resolution } \\
\text { degradation (mm) }\end{array}$ & 60 & Negligible & 20 \\
$\begin{array}{l}\text { Vertical resolution } \\
\text { degradation (mm) }\end{array}$ & 40 & Negligible & 50 \\
\hline
\end{tabular}

The focusing algorithm described in Section 1.2 assumes that the ground is flat. When calculating $T_{i}$, departures from flat ground will therefore, degrade the focusing process. The effects of surface curvature or gradients, discussed in Section 2.6, were therefore investigated by both ray-tracing and FDTD simulations of a $4 \times 72$-D array, for a range of surface profiles and ground parameters. Results (consistent for both methods) are summarised in Table 2; it can be seen that the effects of these ground profiles are relatively small errors in the target location, and a loss of processing gain of up to $4 \mathrm{~dB}$.

Indoor experiments have included successful detection of plastic mine simulants buried in sand in the presence of metal clutter objects $(1563 \mathrm{~cm}$ square pieces of aluminium distributed in the potential elutter volume).

\subsection{Outdoor measurements}

Recently, an experimental system consisting of $4 \times 7$ elements has been deployed in a variety of outdoor environments, in three main sites, where a large number of measurements have been made, of which there is only space here for a small but representative sample. Note that this experimental array is smaller than the full-size system envisaged and hence has lower anticlutter performance and lower, and anisotropic, resolution.

Firstly, targets were buried in damp crushed limestone rock, as used for rough tracks and hence a common environment for land-mines. Fig. 8 shows typical results. The focussed data clearly shows the true positions of a $10 \mathrm{~cm}$ diameter plastic AP mine simulant and $15 \mathrm{~cm}$ diameter metal sphere, both are resolved clearly and distinctly, despite their proximity and their very different reflectivities.

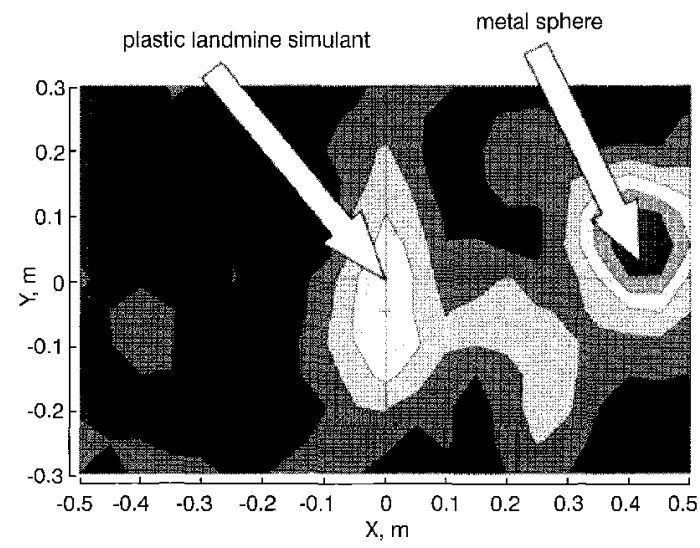

Fig. 8 Plastic antipersonnel landmine (cenire) and metal sphere buried at $0.1 \mathrm{~m}$ (plan view)

IEE Proc.-Radar, Sonar Navig., Vol. 148, No. 4, August 2001 
One particularly challenging situation is where a plastic mine is buried with its upper surface flush with the ground surface. Fig. 9, however, demonstrates successful detection and localisation of a plastic AP mine in exactly this scenario. Fig. 10 further demonstrates detection of a small flush-buried mine simultaneously with that of an anti-tank (AT) mine buried at a depth of $0.5 \mathrm{~m}$.

A second environment considered has been agricultural land (a field in Somerset) where targets were buried in unprepared, moist, loamy soil (which contained stones, roots, etc). Fig. 11 shows typical results for a plastic AP

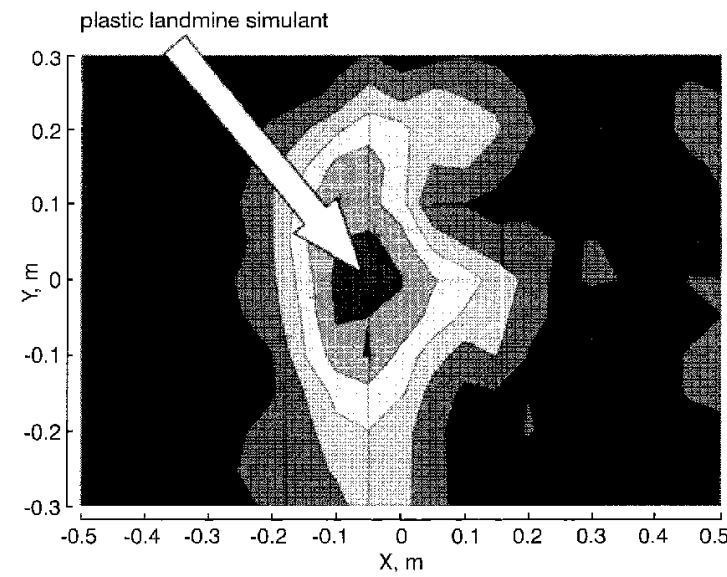

Fig. 9 Flush-buried plastic AP simulant buried at $x=0.0 \mathrm{~m}, y=0.0 \mathrm{~m}$ (plan view)

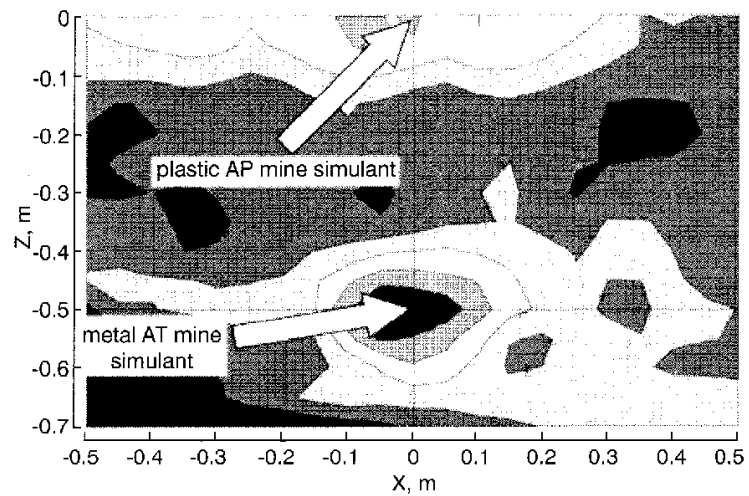

Fig. 10 Flush-huried $A P$ simulant at $x=0.0$, and $A T$ simulant at $x=0.0$ and a depth of $0.5 \mathrm{~m}$ (vertical cross-section)

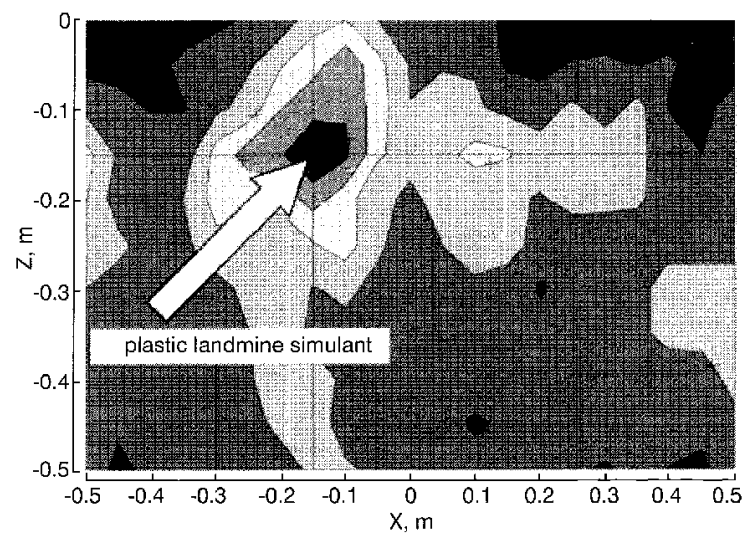

Fig. 11 Plastic antipersonnel land-mine buried at a depth of $0.15 \mathrm{~m}$ $x=-0.15 \mathrm{~m}$ (vertical cross-section) simulant buried at a depth of $15 \mathrm{~cm}$; this is again clearly detected.

Future contributions will present a larger set of results. Further work will include testing the system against targets in other soil types and, ultimately, characterising its performance in terms of detection probabilities and false alarm rates.

\section{Conclusions}

RASOR permits optimum weighting for all distinct paths from transmit to receive clements via any point of interest. This results in significant gains compared to alternative systems, against thermal noise and, morc particularly, against the various forms of clutter. It can also cope with a wide range of surface deformations.

The scheme yields high processing gain and good resolution, independent of soil type and can operate up to high vehicle speeds. With time-sharing, a single transmitter, a single receiver and possibly a single sample-andhold circuit, and a common time-varying gain unit and digitiscr can accomplish all data collection at full operational speed.

Any GPR system will find it difficult to distinguish between a mine and a clutter object of similar size, shape and diclcctric constant. Attempts have been made to identify mine signatures by cataloguing their resonances but this is made difficult by the influenee of differing soil types and target depths on these charactcristics $[16,17]$ ). Fusion with classification data from sensors of complcmentary modality would therefore probably always be desirable in an operational system. The RASOR technique, however, appears to offer an excellent choice for the primary GPR component of such a composite system.

\section{Acknowledgments}

The authors thank DERA Malvern for sponsoring this work and Racal Defence Electronics for providing experimental facilities.

\section{References}

1 US DEPARTMENT OF STATE: 'Hidden killers: the global landmine crisis'. Report to US Congress, 1994

2 HABIGER, K.W., CLIFFORD, J.R., MILLER, R.B., and McCULLOUGI, W.F.: 'Explosives detection with energetic photons', Nuct. Instr. Meth. Phys. Res. B, Beam Interact. Mater. At., 1991, 56/57, pp. 834838

3 CARTER I SULLIVAN M HUNG Y and TENG IC C 'Thermal imaging for landmine detection'. Proceedings of IEE/EUREI conference on the detection of abandoned land-mines, Edinburgh, 1998, pp. $110-114$

4 ADERIN, M.E., and BURCH, I.A.: 'Countermine: hand-held and vehicle mounted mine detection'. Proceedings of IEE/EUREL conference on the detection of abandoned land-mines, Edinburgh, 1998, pp. $198-202$

5 DANIELS, D. : 'Surface penctrating radar' (IEE, UK, 1998)

6 MAGG, M.: 'Mine detection with microwaves'. RTO Lecture Series 214 (Advances in Pattern Recognition Techniques), NATO Research and Technology Organisation, 1998, 8, pp. 1-12

7 BENJAMIN, R.: 'Post-reception focusing in remote detection systems' US patent $5920285,6 / 7 / 99$

8 BENJAMIN, R . 'Post-detection synthetic focusing in near-field radar'. IEE/EUREL conference on the detection of abandoned land-mines, Edinburgh, 1996, pp. 133-137

9 BENJAMIN, R., HILTON, G.S., NIL $A$ VLL $\Lambda$ N, R., LITOBARSKI, S. and MCCUTCHEON, E.: 'Synthetically-focused surface-penetrating radar for operation from a moving vehicle'. IEE conference on the detection of abandoned land-mines, fdinburgh, 1998, pp. 6063

10 BENJAMIN, R. HULTON GS, LITOBARSKI, S, McCUTCHEON, E., and NILAVALAN, R.: 'Post-detection synthetic near-ficld focusing in radar or sonar', Electron. Lett., 1999, 35. (8), pp. 664666

11 EIDE, E.S., and H.JELMSTAD, J.F.: 'The development of an advanced mine detection system at the Norwegian University of Science and Technology'. Proceedings of IEF/EUREL conference on the detection of abandoned land-mines, Edinburgh, 1998, pp. 101-104 
12 BENJAMN, R.: 'Synthetic-aperture antennas', Microwave J., 1995, 38

13 KINGSLEY, S., and QUEGAN, S.: 'Understanding radar systems' (McGraw-Hill, 1992), p. 263

14 NILAVALAN, R., HILTON, G.S., and BENJAMIN, R.: " $A$ FDTD model for the post-detection synthetic focusing surface-penetrating radar with mine-detection applications'. IFF/URSI conference on antennas and propagation, 1999 , pp. 69-72

15 NILAVALAN, R., HILTON, G.S., and BENJAMIN, R.: 'Wideband printed bowtic antenna element development for post reception synthetic focussing surface penetrating radar', Electron. Lett, 1999, 35, (20), pp. 1771-1772

16 PETERS, L., DANIELS, J.J., and YOUNG, J.D.: 'Ground penetrating radar as an environmental sensing tool', Proc. IEEE, 1994, 82, pp. 1802-1822

17 VITEBSKIY, S., and CARIN, L: 'Resonances of perfectly conducting wires and bodies of revolution buried in a lossy, dispersive half-space" IEEE Trans. Antennas Propag., 1996, 28, pp. 1575-1583 